\title{
Synthesis of ZnO Nanowire Heterostructures by Laser Ablation and Their Photoluminescence
}

\author{
Daisuke NAKAMURA, Takafumi MATSUMOTO, Akio KUMEDA, Kazuyuki TOYA, Kota OKAZAKI, \\ Mitsuhiro HIGASHIHATA and Tatsuo OKADA \\ Department of Electrical Engineering, ISEE, Kyushu University \\ 744 Motooka, Nishi-ku, Fukuoka819-0395, Japan \\ E-mail: dnakamura@ees.kyushu-u.ac.jp
}

\begin{abstract}
$\mathrm{ZnO}$ nano-crystals have been paid a great attention as building blocks for the optoelectronic devices, such as an UV-LED. We have been succeeded in growing $\mathrm{ZnO}$ nanostructures, such as vertically-aligned $\mathrm{ZnO}$ nanowires and nanowalls, by a newly developed nanoparticle-assisted pulsedlaser deposition without using any catalyst. Depending on the growth condition a film-wire layerstructured $\mathrm{ZnO}$ and core/shell structured $\mathrm{ZnO}$ nanowires were synthesized on sapphire substrates. The room temperature photoluminescence spectrum of synthesized $\mathrm{ZnO}$ nanostructures exhibited an intrinsic UV emission and a defect-related visible emission.
\end{abstract}

DOI:10.2961/jlmn.2011.01.0006

Keywords: ZnO, PLD, Nanowire, Layered-structure, Optoelectronic devices

\section{Introduction}

Zinc oxide $(\mathrm{ZnO})$, which has a direct wide band-gap of $3.37 \mathrm{eV}$ at room temperature and a relatively large exciton binding energy of $60 \mathrm{meV}$, is one of the promising materials in UV optoelectronic applications such as a lightemitting diode (LED) and a laser diode (LD). In addition, since $\mathrm{ZnO}$ has a strong tendency for self-organized growth, $\mathrm{ZnO}$ nanostructures could be synthesized using some such methods as a chemical vapor deposition [1] and a nanoparticle-assisted pulsed-laser deposition (NAPLD) [2,3]. Especially, ZnO nanowire has attracted a great attention for building blocks of nanodevices such as an UV-LD, an UVLED [4-8], and an UV gas sensor [9,10], because it has superior crystalline quality, better electrical/optical quality, freedom to choose substrate and large surface area to volume ratio. Furthermore, $\mathrm{ZnO}$ nanowires have no need for a lattice matched substrate for the overgrowth [11].

For the practical optoelectronic applications based on the $\mathrm{ZnO}$ nanowires, however, three important issues are essentially required: p-type doping, growth control and fabrication of layered structures for $\mathrm{p}-\mathrm{n}$ junction and coreshell structure. In our study, we have succeeded in synthesizing various nanostructures, such as nanorods [1215], nanowalls [16], the vertically and horizontally aligned $\mathrm{ZnO}$ nanowires $[17,18]$ by NAPLD without any catalyst. Recently, we found that $\mathrm{ZnO}$ film and nanowires were synthesized in one chamber with changing the deposition condition. This predicts the $\mathrm{ZnO}$ nanowire layered structures, such as film-wire structure and core/shell structure, can be fabricated in a simple experimental setup. In this paper, we describe progresses of synthesis of layerstructured $\mathrm{ZnO}$ nanowires by NAPLD using a multi-target changer system. In the use of the multi-target changer system, ZnO source targets can be changed without disturbing chamber condition during synthesis of $\mathrm{ZnO}$ nanowires.

\section{Experiments}

Fig. 1 shows the schematic of the experimental setup for synthesis of layer-structured $\mathrm{ZnO}$ nanowires by NAPLD. In the experiment, sintered cylindrical $\mathrm{ZnO}$ source targets were used in synthesizing $\mathrm{ZnO}$ nanowires. A c-plane sapphire substrate $(1 \mathrm{~cm} \times 1 \mathrm{~cm})$ was put on a $\mathrm{SiC}$ heater in a vacuum chamber and the target-substrate distance was set to $40 \mathrm{~mm}$. The substrate was heated to $400-800{ }^{\circ} \mathrm{C}$ in the vacuum chamber filled with a background gas of argon or

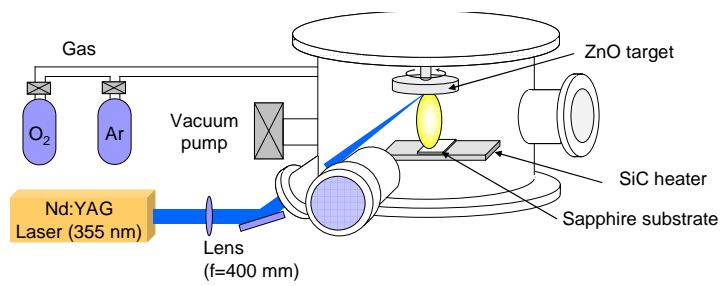

Fig. 1 Schematic of the experimental setup for synthesis of layer-structured $\mathrm{ZnO}$ nanowires by NAPLD.

oxygen. The $\mathrm{ZnO}$ target was ablated with third harmonic generation (THG) of a Q-switched Nd:YAG laser at 355 $\mathrm{nm}$ with a repetition rate of $10 \mathrm{~Hz}$ and a fluence of about $1.3 \mathrm{~J} / \mathrm{cm}^{2}$. The morphology of the as-deposited products was analyzed by scanning electron microscopy (SEM). The optical properties of the $\mathrm{ZnO}$ nanowires were investigated by observing the photoluminescence (PL) with THG of other Q-switched Nd:YAG laser.

\section{Results and Discussion}

\subsection{Synthesis of $\mathrm{ZnO}$ nanowires}

In NAPLD, ZnO nanostructures are synthesized at a higher background gas pressure. A sintered $\mathrm{ZnO}$ target with 99.99\% purity was used as source material in synthesizing $\mathrm{ZnO}$ nanowires. Fig.2 shows the SEM image of the $\mathrm{ZnO}$ nanowires on the c-plane sapphire substrate at $800{ }^{\circ} \mathrm{C}$ and 


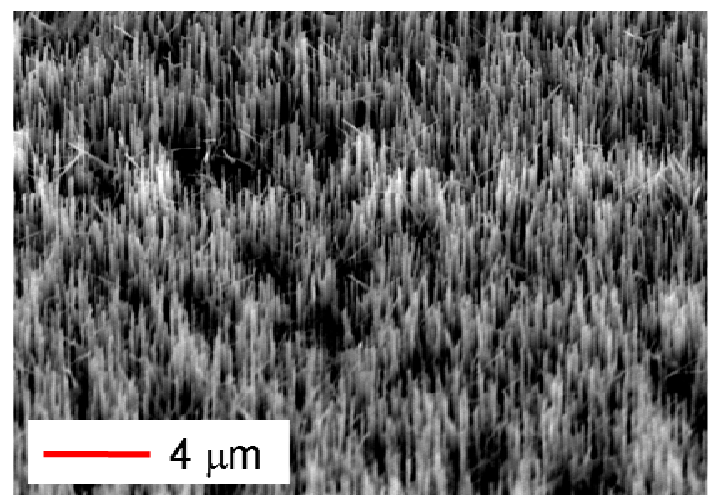

Fig. 2 SEM image ( $45^{\circ}$ tilted view) of $\mathrm{ZnO}$ nanowires.

background argon gas pressure of 200 Torr. In NAPLD, the ablated species were perfectly confined in a small volume with a stoichiometric composition of $\mathrm{ZnO}$, and then $\mathrm{ZnO}$ nanoparticles are formed in the gas phase during the transport of the ablated species to the substrate. Nanoparticles deposited on the substrate form thin buffer layer and some nucleation. Then nanowires grow on them. It can be seen the vertically-aligned thin $\mathrm{ZnO}$ nanowires with average length of about $2 \mu \mathrm{m}$.

\subsection{Film-Wire Layer-Structured ZnO Nanowires}

As an initial demonstration of fabricating the film-wire layered structure, $\mathrm{ZnO}$ nanowires were synthesized after deposit a ZnO film. The film was deposited on a c-plane
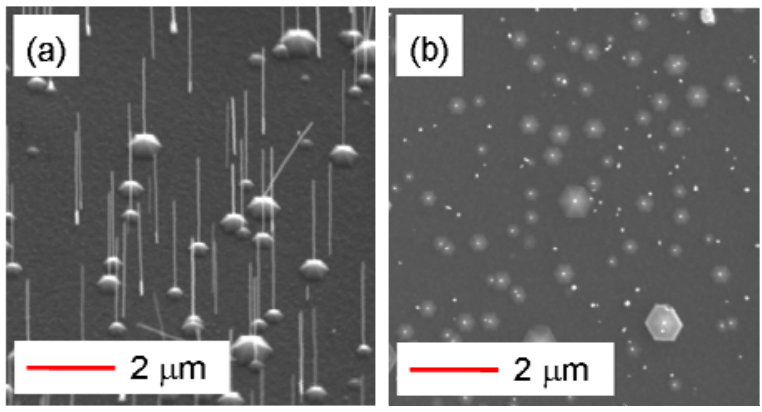

Fig. 3 SEM images of $\mathrm{ZnO}$ nanowires synthesized on the $\mathrm{ZnO}$ film, (a) $45^{\circ}$ tilted view and (b) top view.

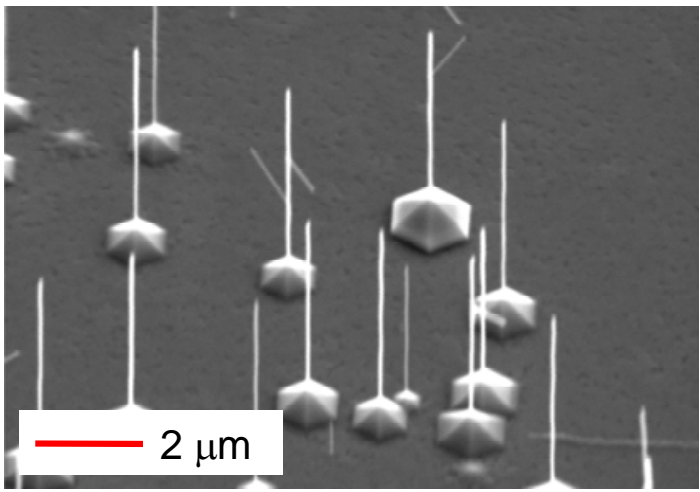

Fig. 4 SEM image of the $\mathrm{ZnO}$ nanowires synthesized on the $\mathrm{Li}$-Ni codoped $\mathrm{ZnO}$ film. sapphire substrate temperature of $650{ }^{\circ} \mathrm{C}$ and oxygen gas pressure of 26 mTorr. After deposition of the ZnO film for 5 minutes, the nanowires were subsequently synthesized on the film at the temperature of $750{ }^{\circ} \mathrm{C}$ and argon gas of 200 Torr in the same chamber. The growth time of the $\mathrm{ZnO}$ nanowires was $15 \mathrm{~min}$. Fig.3 shows the SEM image of the $\mathrm{ZnO}$ nanowires on the film. It was found that the verticallyaligned low density $\mathrm{ZnO}$ nanowires with the diameter of $50-100 \mathrm{~nm}$ and the length of around $1.5 \mu \mathrm{m}$. Most nanowires were grown on the hexagonal pyramid. This result is similar to that of Cao et al. [19]. They say that $\mathrm{ZnO}$ buffer film seems to prevent nanowire nucleation and growth since the incoming $\mathrm{ZnO}$ incorporates much more effectively into the $\mathrm{ZnO}$ buffer film. Although the more detail mechanism should be investigated, an interaction between the buffer film and the depositing nanoparticles occurred.

Furthermore, layer-structured $\mathrm{ZnO}$ nanowires consisting of different material layers also could be synthesized using a non-doped and a Li-Ni codoped ZnO targets. Fig.4 shows the SEM image of the $\mathrm{ZnO}$ nanowires synthesized on the Li-Ni codoped $\mathrm{ZnO}$ film. The growth condition was the same as in Fig. 3. The vertically-aligned low density $\mathrm{ZnO}$ nanowires were also synthesized on the $\mathrm{Li}-\mathrm{Ni}$ codoped $\mathrm{ZnO}$ film. This indicates the film-wire layered structure consisting of different $\mathrm{ZnO}$ targets is fabricated by the multi-target changer system in the simple experimental setup.

\subsection{Core/Shell Structured ZnO Nanowires}

It is important to fabricate layered structure in not only the nanowire axial direction but also the radial direction, because core/shell nanowires are expected to be more efficient in emitting due to low thermal quenching and large surface effect.

Fig.5(a) shows the SEM image of the $\mathrm{ZnO}$ nanowires on the film after growth of the $\mathrm{ZnO}$ core at the same deposition condition as Fig. 3 and 4. Fig. 5(b),(c) show the top view and $45^{\circ}$ tilted view SEM images of $\mathrm{ZnO}$ nanowires
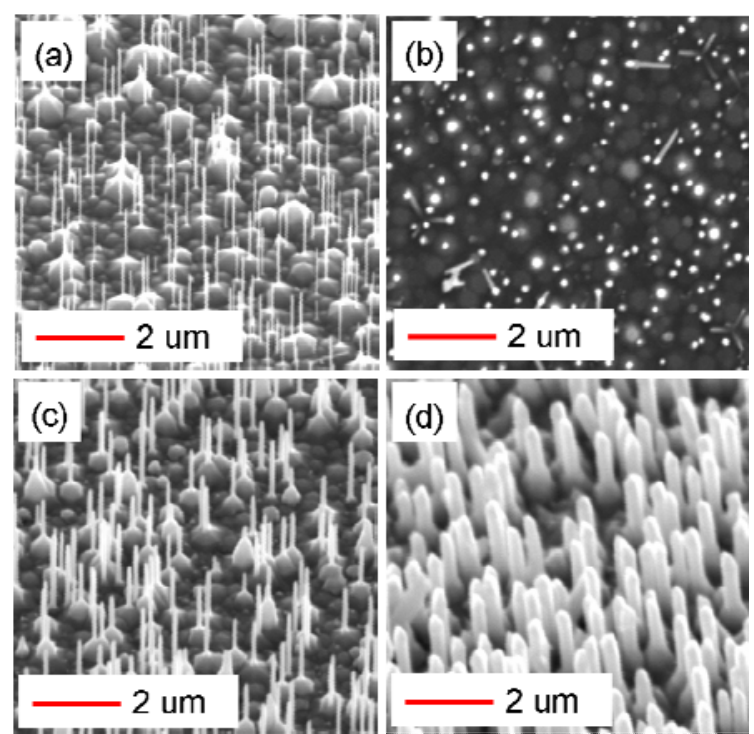

Fig. 5 SEM images of (a) ZnO nanowires on the film and core/shell structued $\mathrm{ZnO}$ nanowires, where the growth time of the shell layer were 5 min ((a) top view, (c) $45^{\circ}$ tilted view), and (d) $20 \mathrm{~min}$. 


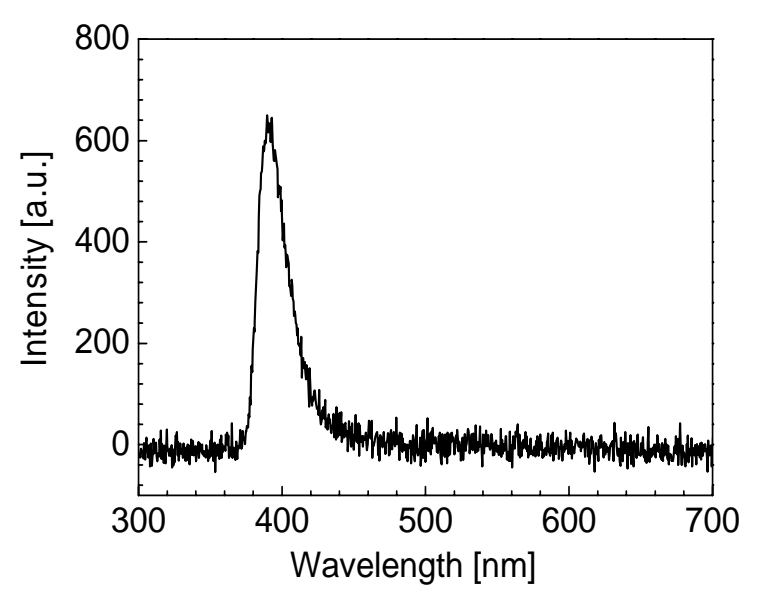

Fig. 6 PL spectrum of the $\mathrm{ZnO}$ nanowires on the $\mathrm{ZnO}$ film.

after growth the shell for 5 minutes at the temperature of $650{ }^{\circ} \mathrm{C}$ and oxygen gas pressure of 26 mTorr. The $\mathrm{ZnO}$ nanowires grew uniformly in the radial direction, indicating that $\mathrm{ZnO}$ shell coats homogeneously the entire nanowire. Fig. 5(d) shows the SEM image of the $\mathrm{ZnO}$ nanowires after growth of the $\mathrm{ZnO}$ shell for 20 minutes. The diameter of the $\mathrm{ZnO}$ nanowire increased uniformly with increasing the deposition time. Thus, it was found that thickness of the shell could be controlled by the deposition time and expected that core/shell structure consisting of different material layers could be fabricated using different kinds of targets.

\subsection{Photoluminescence of the $\mathrm{ZnO}$ nanowires}

The photoluminescence characteristic of the asdeposited products was also investigated. Fig. 6 shows the $\mathrm{PL}$ spectrum of the $\mathrm{ZnO}$ nanowires on the $\mathrm{ZnO}$ film shown in Fig. 3 by spectrometer (Lambda Vision, LVM200-KS), where the power of the excitation laser was $90 \mathrm{~kW} / \mathrm{cm}^{2}$ and the exposure time was 2 sec. The UV emission centered at around $390 \mathrm{~nm}$, which is the contribution of the near band edge emission of the wide band-gap $\mathrm{ZnO}$, and no visible light emission at green emission band attributed to the deep-level defects inside $\mathrm{ZnO}$ lattices were observed. The deep-level luminescence is related to the oxygen vacancies in the grown $\mathrm{ZnO}$ crystals. This indicates a very low concentration of deep-level defects inside $\mathrm{ZnO}$ lattices.

\section{Conclusions}

To summarize, we have succeeded in synthesizing vertically aligned $\mathrm{ZnO}$ nanorods, nanowires on c-plane sapphire substrates by NAPLD in the same chamber. In this study, the film-wire layer-structured $\mathrm{ZnO}$ nanowires were synthesized. Vertically-aligned low density $\mathrm{ZnO}$ nanowires with were synthesized on the $\mathrm{ZnO}$ film. In addition, synthesis of the film-wire layered structure $\mathrm{ZnO}$ nanowires consist- ing of different $\mathrm{ZnO}$ targets were demonstrated. Core/shell structured $\mathrm{ZnO}$ nanowires were also fabricated in the single chamber. Furthermore, near band edge emission of $\mathrm{ZnO}$ was observed from synthesized $\mathrm{ZnO}$ nanowires.

\section{Acknowledgments}

A part of this study has been financially supported by Special Coordination Funds for Promoting Science and Technology from Japan Science and Technology Agency and a Grant-in Aid for Scientific Research from the Japan Society of Promotion of Science.

\section{References}

[1] J.-J. Wu, S.-C. Liu: Adv. Mater., 14, (2002) 215.

[2] A. B. Hartanto, X. Ning, Y. Nakata, T. Okada: Appl. Phys. A, 78, (2004) 299.

[3] T. Okada, A. B. Hartanto, Y. Nakata: Appl. Phys. A, 79, (2004) 1417.

[4] R. Guo, J. Nishimura, M. Matsumoto, M. Higashihata, D. Nakamura, T. Okada: Appl. Phys B, 94, (2009) 33.

[5] M.-C. Jeong, B.-Y. Oh, M.-H. Ham, J.-M. Myounga: Appl. Phys. Lett., 88, 202105 (2006).

[6] H. Gao, F. Yan, J. Li, Y. Zeng, J. Wang: J. Phys. D: Appl. Phys., 40, (2007) 3654.

[7] Y. Yang, X. W. Sun, B. K. Tay, G. F. You, S. T. Tan, K. L. Teo: Appl. Phys. Lett. 93, (2008) 253107.

[8] M. Kumar, J. P. Kar, I.-S. Kim, S.-Y. Choi, J.-M. Myoung: Appl Phys A, 97, (2009) 689.

[9] J. Suehiro, N. Nakagawa, S. Hidaka, M. Ueda, K. Imasaka, M. Higashihata, T. Okada, M. Hara: Nanotechnology, 17, (2006) 2567.

[10] T. Okada, J. Suehiro: Appl. Sur. Sci., 253, (2007) 7840.

[11] Willander M, Yang L L, Wadeasa A, Ali S U, Asif M H, Zhao Q X and Nur O: J. Mater. Chem. 19, (2009) 1006.

[12] T. Okada, K. Kawashima, M. Ueda: Appl. Phys. A, 81, (2005) 907.

[13] M. Kawakami, A. B. Hartanto, Y. Nakata, T. Okada: Jpn. J. Appl. Phys., 42, (2003) L33.

[14] T. Okada, K. Kawashima, Y. Nakata, X. Ning: Jpn. J. Appl. Phys., 44, (2005) 688.

[15] T. Okada, K. Kawashima, Y. Nakata: Thin Solid Films, 274, (2006) 506.

[16]B. Q. Cao, T. Matsumoto, M. Matsumoto, M. Higashihata, D. Nakamura, T. Okada: J. Phys. Chem. C, 113, (2009) 10975.

[17] R. Q. Guo, J. Nishimura, M. Ueda, M. Higashihata, D. Nakamura, T. Okada: Appl. Phys. A, 89, (2007) 141.

[18]R. Guo, M. Matsumoto, T. Matsumoto, M. Higashihata, D. Nakamura, T. Okada: Appl. Sur. Sci., 255, (2009) 9671.

[19] R. Q. Guo, J. Nishimura, M. Matsumoto, M. Higashihata, D. Nakamura, T. Okada: Jpn. J. Appl. Phys., 47, (2008) 741. 